\title{
Identifying a potential tool to measure household food insecurity in the UK: a systematic review
}

\author{
G. Nguyen ${ }^{1}$, L. Aucott ${ }^{1}$, G. McNeill ${ }^{1}$ and F. Douglas ${ }^{2}$ \\ ${ }^{1}$ The Institute of Applied Health Sciences and \\ ${ }^{2}$ The Rowett Institute, University of Aberdeen, Scotland, AB25 2ZD, UK
}

The growth of high-profile food aid initiatives and number of people seeking food bank support in recent years has raised awareness of household food insecurity (HFI) in the UK. The original work in this area identified four domains of HFI: food quantity, food quality, psychological and social dimensions ${ }^{(1)}$. HFI was first assessed in the UK in the Low Income Diet and Nutrition Survey (2003$2005)^{(2)}$ and more recently in the Gallup World Poll in 2014, in which the prevalence of HFI was $10 \%$, and therefore accounted for 8.4 million people in the $\mathrm{UK}^{(3)}$. Various survey tools have been developed to measure the experience of HFI in developed countries like Canada and the USA. The data can be used to describe the prevalence, risk factors and consequences of HFI and to monitor the effectiveness of policies which aim to tackle this issue. However, at present no consensus exists on the best HFI measurement for use in the UK. This study aim is to identify the measurement instruments which have been used to measure HFI in developed /high income countries which would be suitable for use in the UK context.

Medline, EMBASE and CINAHL were systematically searched using a comprehensive search strategy which focused on three main facets: food insecurity, measurement and developed/high economy countries (as defined by the World Bank ${ }^{(4)}$ ). The search was limited to English language and to studies conducted between 1996-2016. All eligible studies were obtained and read in their abstract or full text form, and the relevant information extracted using a data extraction form. The findings in relation to which domains of HFI had been included, how the HFI measurement had taken place and the purpose of data collection were recorded and summarised.

From a total of 2,070 articles retrieved after removing duplication from the three databases, 572 records were considered potentially eligible for the data extraction and 451 publications were actually used for the final analysis. Twenty different tools were identified with the most commonly-used being the USDA Household Food Security Survey Module (HFSSM) (66\%), the Canadian HFSSM (8\%). Other tools such as the Radimer/Cornell Hunger Scale, FAO Food Insecurity Experience Scale (FIES) etc. had only been used in a small proportion of the included studies. The majority of the tools had been used to measure HFI in adults (44\%) and/ or mother and child pair (12\%). More than $80 \%$ of the included studies examined the association between HFI and other demographic, health or social variables. Although all four domains of HFI were included in most of the identified tools, information on the psychological and social domains of HFI was less-well captured than that on the food quantity and food quality domains.

Although there are a number of structured scales available in the literature for measuring HFI, the USDA HFSSM stands out as the most commonly used tool in high income countries. Additional work to develop questions to better capture the psychological and social components of HFI is required.

1. Tarasuk (2001) Discussion paper on household food security. http://citeseerx.ist.psu.edu/viewdoc/download?doi=10.1.1.467.3418\&rep=rep1\& type $=$ pdf (accessed April 2017).

2. Nelson M, Ehrens B, Bates B, Church S \& Boshier T (2007) Low Income Diet and Nutrition Survey. Vol 3: Nutritional Status Physical Activity Economic, Social and Other Factors. London: TheStationery Office.

3. Food and Agriculture Organization of the United Nations, Voices of the Hungry (2016). www.fao.org/3/a-i4830e.pdf (accessed April 2017).

4. World Bank: countries classification. https://datahelpdesk.worldbank.org/knowledgebase/articles/906519\#High_income (accessed April 2017). 